\title{
Development of an S-1 dosage formula based on renal function by a prospective pharmacokinetic study
}

\author{
Eisuke Booka $^{1}$ - Chiyo K. Imamura ${ }^{2}$ Hiroya Takeuchi ${ }^{1}$ ' Yasuo Hamamoto ${ }^{3}$ • \\ Daisuke Gomi $^{4}$ - Takuro Mizukami ${ }^{5}$. Takashi Ichiyama ${ }^{6} \cdot$ Kazunari Tateishi $^{6}$. \\ Tsunehiro Takahashi $^{1,3}$ - Hirofumi Kawakubo ${ }^{1}$ Kenzo Soejima ${ }^{7}$ Narikazu Boku ${ }^{5}$. \\ Yusuke Tanigawara $^{2} \cdot$ Yuko Kitagawa $^{1,3}$
}

Received: 21 July 2015/ Accepted: 7 August 2015/Published online: 25 August 2015

(C) The Author(s) 2015. This article is published with open access at Springerlink.com

\begin{abstract}
Background S-1 is an oral anticancer drug, containing tegafur (a prodrug of 5-fluorouracil, 5-FU), 5-chloro-2,4dihydroxypyridine, and potassium oxonate. As renal dysfunction is known to increase exposure of 5-FU following S-1 administration, the incidence of severe adverse reactions is increased in patients with impaired renal function. However, no reliable information on its
\end{abstract}

Electronic supplementary material The online version of this article (doi:10.1007/s10120-015-0536-6) contains supplementary material, which is available to authorized users.

\section{Chiyo K. Imamura}

imamurack@z3.keio.jp

1 Department of Surgery, Keio University School of Medicine, 35 Shinanomachi, Shinjuku-ku, Tokyo 160-8582, Japan

2 Department of Clinical Pharmacokinetics and Pharmacodynamics, School of Medicine, Keio University, 35 Shinanomachi, Shinjuku-ku, Tokyo 160-8582, Japan

3 Keio Cancer Center, Keio University Hospital, 35 Shinanomachi, Shinjuku-ku, Tokyo 160-8582, Japan

4 Department of Comprehensive Cancer Therapy, Shinshu University School of Medicine, 3-1-1 Asahi, Matsumoto, Nagano 390-8621, Japan

5 Department of Clinical Oncology, St. Marianna University School of Medicine, 2-16-1 Sugao, Miyamae-ku, Kawasaki, Kanagawa 216-8511, Japan

6 First Department of Internal Medicine, Shinshu University School of Medicine, 3-1-1 Asahi, Matsumoto, Nagano 390-8621, Japan

7 Division of Pulmonary Medicine, Department of Medicine, Keio University School of Medicine, 35 Shinanomachi, Shinjuku-ku, Tokyo 160-8582, Japan dose modification for patients with renal dysfunction has been provided.

Methods We conducted a prospective pharmacokinetic study to develop an S-1 dosage formula based on renal function. Sixteen cancer patients with various degrees of renal function received a single dose of S-1 at $40 \mathrm{mg} / \mathrm{m}^{2}$. A series of blood samples were collected at predefined times within $24 \mathrm{~h}$ to assess the plasma concentration profiles of 5-FU, 5-chloro-2,4-dihydroxypyridine, and tegafur. A mathematical model for the relationship between renal function and exposure of 5-FU was constructed by a population pharmacokinetic analysis.

Results The clearance of 5-FU following S-1 administration was related to body surface area and creatinine clearance in the range $15.9-108.8 \mathrm{~mL} / \mathrm{min}$ as estimated by the Cockcroft-Gault equation. The S-1 dosage formula was derived as follows:

dose $=$ target AUC $\times(21.9+0.375 \times$ CLcr $) \times$ BSA,

where AUC is the area under the concentration-time curve, CLcr is creatinine clearance, and BSA is body surface area. The recommended daily doses of S-1 in Asia and Europe were also proposed as nomograms according to exposure matching to the previously reported area under the concentration-time curve of 5-FU, which confirmed the efficacy and toxicity in pivotal registration studies.

Conclusions We have developed a novel formula for determining the S-1 dosage on the basis of renal function. Further validation is needed to confirm the formula for practical application.

Keywords Creatinine clearance - Dosage formula . Population pharmacokinetics $\cdot$ Renal function $\cdot$ S-1 


\section{Introduction}

After entering the body, a drug is eliminated by metabolism and/or excretion. Although elimination can occur via several routes, most drugs are cleared by metabolism in the liver and/or elimination through the kidney. For a drug eliminated primarily via renal excretory mechanisms, impaired renal function alters its pharmacokinetics to an extent that its dosage needs to be changed from that used in patients with normal renal function. The US Food and Drug Administration [1] and the European Medicines Agency [2] recommend that the pharmacokinetics of a new drug be assessed in patients with impaired renal function during the development phase and that rational dosing recommendations be provided. However, there is no similar guidance in Japan; thus, dose modification for patients with renal dysfunction is based on data from the USA and Europe.

Since Heidelberger et al. [3] reported 5-fluorouracil (5FU) in 1957, 5-FU has been a key drug for gastrointestinal tumors. An oral drug S-1 was first approved in Japan in 1999 and is widely used in Asia and Europe (TS- $1^{\circledR}$, Taiho Pharmaceutical, Tokyo, Japan, in Asia; Teysuno ${ }^{\circledR}$, Nordic Group, Hoofddrop, The Netherlands, in Europe). S-1 contains tegafur, which is a prodrug of 5-FU, 5-chloro-2,4dihydroxypyridine (CDHP), which inhibits the rate-limiting enzyme (dihydropyrimidine dehydrogenase) of 5-FU catabolism to maintain a high concentration of 5-FU, and potassium oxonate, which is specifically distributed in the epithelium of the intestine and inhibits the phosphorylation of 5-FU to reduce gastrointestinal toxicity [4-6] (Fig. 1). The approved dosage and administration of S-1 are $80 \mathrm{mg} /$ $\mathrm{m}^{2} /$ day as tegafur for 4 weeks followed by 2 weeks of rest in Asia [7, 8], and $50 \mathrm{mg} / \mathrm{m}^{2} /$ day as tegafur for 3 weeks followed by 1 week of rest in combination with cisplatin at $75 \mathrm{mg} / \mathrm{m}^{2}$ once every 4 weeks in Europe [9]. S-1 is a standard adjuvant treatment for East Asian patients who have undergone a D2 dissection for locally advanced gastric cancer [10] and it is noninferior to 5-FU for patients with metastatic gastric cancer [11]. S-1 in combination with cisplatin is a standard first-line treatment for patients with advanced gastric cancer [12].

Since more than $50 \%$ of CDHP is excreted in urine [13], renal dysfunction increases exposure of CDHP, and results in a sustained high concentration of 5-FU [14]. The results of a postmarketing survey of S-1 administered at $80 \mathrm{mg} / \mathrm{m}^{2} /$ day as tegafur revealed that the incidence of grade 3 or grade 4 adverse reactions increased in patients with impaired renal function. S-1 is contraindicated in patients with severe renal dysfunction, but no information on dose modification for patients with mild and moderate renal dysfunction is provided on the Asian package inserts $[7,8]$.

We therefore attempted to develop an S-1 dosage formula based on renal function to determine the recommended dose for patients with impaired renal function. A prospective pharmacokinetic study was conducted to assess the plasma concentration profiles of 5-FU, CDHP, and tegafur in patients with various degrees of renal function. A mathematical model for the relationship between renal function and exposure of 5-FU was constructed by a population pharmacokinetic (PPK) analysis.

\section{Patients and methods}

\section{Patient eligibility}

The eligibility criteria of this study were as follows: histologically confirmed solid tumor in patients for whom administration of S-1 was planned; creatinine clearance (CLcr) estimated by the Cockcroft-Gault equation [15] of $15 \mathrm{~mL} / \mathrm{min}$ or greater; age 20 years or older; Eastern

\section{S-1}

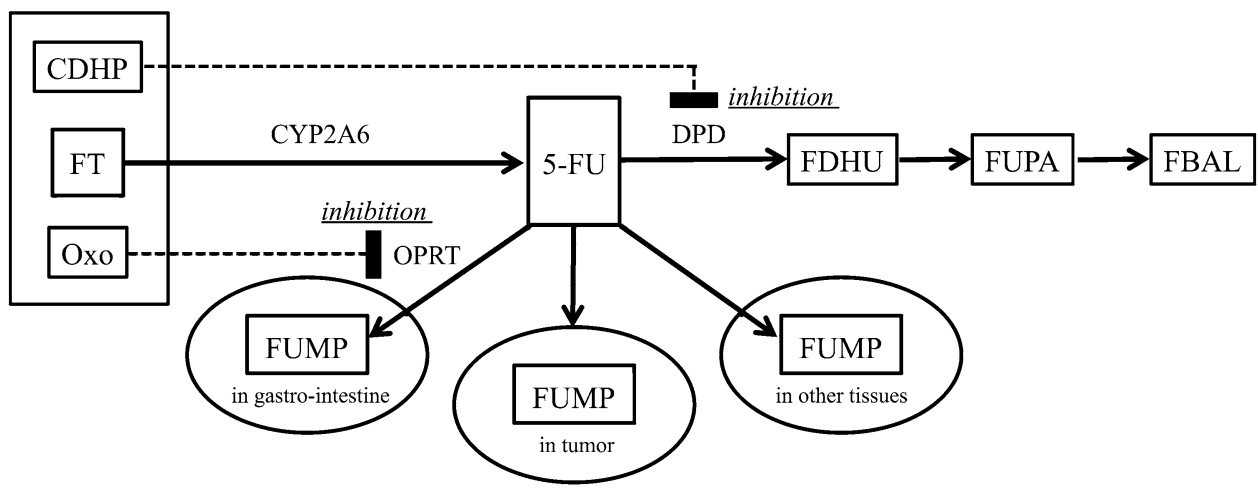

Fig. 1 Biochemical action of S-1. CDHP 5-chloro-2,4-dihydroxypyridine, $C Y P 2 A 6$ cytochrome $\mathrm{P} 4502 \mathrm{~A} 6, D P D$ dihydropyrimidine dehydrogenase, $F B A L \alpha$-fluoro- $\beta$-alanine, $F D H U$ fluorodihydrouracil,
$F T$ tegafur, 5-FU 5-fluorouracil, FUMP fluorouridine monophosphate, FUPA $\alpha$-fluoro- $\beta$-ureidopropionic acid, OPRT orotate phosphoribosyltransferase, Oxo potassium oxonate 
Cooperative Oncology Group performance status 0-2; adequate organ function except renal function (white blood cell count $3000-12,000 / \mathrm{mm}^{3}$; neutrophil count $1500 / \mathrm{mm}^{3}$ or greater; platelet count $100,000 / \mathrm{mm}^{3}$ or greater; total bilirubin concentration $2.0 \mathrm{mg} / \mathrm{dL}$ or lower; aspartate transaminase and alanine transaminase concentration $100 \mathrm{U} / \mathrm{L}$ or lower); ability to take medications orally; and no previous administration of S-1 within 14 days if used.

The study was performed as a project of the Promotion Plan for the Platform of Human Resource Development for Cancer by the Ministry of Education, Culture, Sports, Science and Technology in Japan. The study protocol was approved by each participating institution's institutional review board and was registered in the University Hospital Medical Information Network Clinical Trials Registry under the number UMIN000011708. The study procedures were in accordance with the ethical standards of the Declaration of Helsinki. Written informed consent was obtained from all patients before their enrollment in the study.

\section{Study design}

This prospective pharmacokinetic study in cancer patients with various degrees of renal function was conducted at three institutions in Japan. The primary endpoint was to assess the pharmacokinetic profiles of 5-FU, CDHP, and tegafur after a single dose of S-1 in patients with various degrees of renal function. The secondary endpoint was to evaluate the toxicity within $24 \mathrm{~h}$ of S-1 administration in the patients. US National Cancer Institute Common Terminology Criteria for Adverse Events version 4.0 was used for the toxicity assessment [16]. The study duration was $24 \mathrm{~h}$ after administration of S-1.

\section{Drug formulation and administration}

S-1 (TS- $\left.1{ }^{\circledR}\right)$ orally disintegrating tablets containing tegafur, CDHP, and potassium oxonate in a molar ratio of 1:0.4:1 were administered to the patients. Two dosages of tegafur were used: 20 and $25 \mathrm{mg}$.

The enrolled patients received a single dose of S-1 at $40 \mathrm{mg} / \mathrm{m}^{2}$ as tegafur, which is the approved regular single dose and half of the daily dose in Asia [7, 8, 10]. S-1 was taken within $30 \mathrm{~min}$ after breakfast at a dose of $40 \mathrm{mg}$ as tegafur (two tablets each containing $20 \mathrm{mg}$ ) for patients with a body surface area (BSA) of less than $1.25 \mathrm{~m}^{2}$, $50 \mathrm{mg}$ as tegafur (two tablets each containing $25 \mathrm{mg}$ ) for those with $1.25 \mathrm{~m}^{2} \leq \mathrm{BSA}<1.50 \mathrm{~m}^{2}$, and $60 \mathrm{mg}$ as tegafur (three tablets each containing $20 \mathrm{mg}$ ) for those with $\mathrm{BSA} \geq 1.50 \mathrm{~m}^{2}$.

\section{Estimation of renal function}

To assess the impact of renal function on the pharmacokinetics of CDHP and 5-FU, two commonly used serumcreatinine-based equations, the Cockcroft-Gault equation and the Japanese glomerular filtration rate (GFR) equation [17], were used to estimate renal function.

\section{Pharmacokinetic sample acquisition and handling}

Blood samples were obtained before and 1, 2, 4, 7, 12, and $24 \mathrm{~h}$ after administration. Peripheral blood samples were drawn into heparinized tubes at a volume of $3 \mathrm{~mL}$ at each sampling time and were centrifuged at $3000 \mathrm{rpm}$ for $10 \mathrm{~min}$ at room temperature. The resulting plasma was frozen and stored at $-20{ }^{\circ} \mathrm{C}$ until analysis.

\section{Determination of the plasma concentrations of 5-FU, CDHP, and tegafur}

The plasma concentrations of 5-FU, CDHP, and tegafur were determined with an ultraperformance liquid chromatography (UPLC)-tandem mass spectrometry method developed specifically for this study by modification of a previously reported method [18]. 5-FU, tegafur, and 5-bromouracil, an internal standard, were purchased from Sigma-Aldrich (St Louis, MO, USA). CDHP was obtained from Toronto Research Chemicals (North York, ON, Canada).

Patient plasma $(100 \mu \mathrm{L})$ or an equivalent volume of plasma that contained known concentrations of 5-FU, CDHP, and tegafur was mixed with $250 \mu \mathrm{L}$ of the internal standard solution (5-bromouracil at $100 \mathrm{ng} / \mathrm{mL}$ in acetonitrile). The mixture was vortexed and centrifuged at $13,000 \mathrm{rpm}$ for $5 \mathrm{~min}$. The resulting supernatant was transferred to a glass tube and evaporated to dryness under air at $40{ }^{\circ} \mathrm{C}$. The dried residue was reconstituted in $75 \mu \mathrm{L}$ of $0.1 \%$ formic acid-acetonitrile (90:10, v/v) and transferred to an autosampler vial tube.

The UPLC-tandem mass spectrometry system was equipped with an Acquity UPLC system and a Xevo TQ mass spectrometer (Waters, Milford, MA, USA). Chromatographic separations were obtained under gradient conditions with an ACQUITY UPLC HSS T3 column $(100 \mathrm{~mm} \times 2.1-\mathrm{mm}$ inner diameter, $1.8-\mu \mathrm{m}$ particle size; Waters). The mobile phase consisted of eluent A (0.1\% formic acid) and eluent $B$ (acetonitrile). The flow rate was $0.3 \mathrm{~mL} / \mathrm{min}$, and the gradient was as follows: $8 \%$ eluent $\mathrm{B}$ for $2 \mathrm{~min}, 95 \%$ eluent $\mathrm{B}$ at $4.5 \mathrm{~min}$, and $8 \%$ eluent $\mathrm{B}$ at $6.6 \mathrm{~min}$. The total run time was 9 min per sample. The column temperature was $40{ }^{\circ} \mathrm{C}$, the sample temperature was $10{ }^{\circ} \mathrm{C}$, and the injection volume was $5 \mu \mathrm{L}$. Under these conditions, the retention times for 5-FU, CDHP, tegafur, 
and 5-bromouracil (internal standard) were 1.07, 2.59, 3.54 , and $1.54 \mathrm{~min}$, respectively.

The mass spectrometer was run in negative electrospray ionization mode for 5-FU and in the positive mode for CDHP and tegafur. The source conditions were as follows: capillary voltage, $3 \mathrm{kV}$; cone voltage, $30 \mathrm{~V}$; and desolvation temperature, $400{ }^{\circ} \mathrm{C}$. A collision gas flow rate of $0.28 \mathrm{~mL} / \mathrm{min}$ and collision energy of $5 \mathrm{keV}$ were used for the creation of daughter ions. The multiple reaction monitoring mode detected the following transitions: $129.1 \rightarrow 41.9$ and $189.0 \rightarrow 41.9$ for 5 -FU and 5-bromouracil, respectively, in the negative mode and $146.1 \rightarrow 72.9,201.2 \rightarrow 131.0$, and $191.1 \rightarrow 117.8$ for CDHP, tegafur, and 5-bromouracil, respectively, in the positive mode. The chromatographic data were acquired and analyzed with MassLynx equipped with QuanLynx (Waters).

The concentrations of 5-FU, CDHP, and tegafur in patient plasma samples were calculated by determining the ratios of the areas of 5-FU, CDHP, and tegafur in each sample to the area of the internal standard in that sample and by comparing these ratios to the standard curve prepared on the same day as the samples. The concentration ranges of the standard curves were $5-500 \mathrm{ng} / \mathrm{mL}$ for $5-\mathrm{FU}$ and CDHP and $25-2500 \mathrm{ng} / \mathrm{mL}$ for tegafur. The interday and intraday variabilities in precision (expressed as the coefficient of variation) for all compounds ranged from 0.3 to $5.8 \%$ and from 4.7 to $8.9 \%$, respectively. The average accuracies for the compounds ranged from 100.7 to $107.6 \%$.

\section{PPK analysis}

The PPK analysis was performed with NONMEM (version 7.2; ICON Development Solutions, Dublin, Ireland) and GNU Fortran compiler version 4.6.0 on Microsoft Windows 7. The basic pharmacokinetic parameters were clearance, volume of distribution, and the first-order absorption rate constant $\left(K_{\mathrm{a}}\right)$. As all doses were given by oral administration, clearance and the volume of distribution were interpreted as the ratio of clearance to bioavailability and the ratio of the volume of distribution to bioavailability, respectively.

As a pharmacokinetic structural model of CDHP, 5-FU, and tegafur, a linear one-compartment model with firstorder absorption (subroutines ADVAN2 and TRANS2) without the absorption lag time was used because it was reported that a one-compartment model was more suitable than a two-compartment model and the introduction of the absorption lag time did not improve the model fitting [19].

The interindividual variability was assumed to obey a log-normal distribution and is described for each parameter as follows: $\theta_{j}=\theta \times \exp \left(\eta_{j}\right)$

where $\eta_{j}$ is the random effect for individual $j, \theta$ is the population mean parameter, and $\eta$ is a random variable with mean zero and variance $\omega^{2}$. Residual variability was described by a proportional error model as follows:

$C_{i, j}=$ Cpred $_{i, j} \exp \left(\varepsilon_{i, j}\right)$,

where $\operatorname{Cpred}_{i, j}$ is the $i$ th model-predicted concentration for patient $j, C_{i, j}$ is the measured concentration, and $\varepsilon_{i, j}$ denotes the residual intraindividual random error.

Demographic variables, such as renal function, patient age, sex, BSA, and history of gastrectomy, were examined to identify whether these variables could explain the observed substantial interindividual variability. Demographic variables were included one at a time by stepwise selection based on the likelihood ratio test. The minimum value of the NONMEM objective function was used as a statistic for choosing suitable models during the modelbuilding process. The potentially significant covariates were identified as those factors that when added to the basic model individually resulted in a decrease in the objective function of 3.84 or more $(p<0.05)$.

To evaluate the validity and robustness of the PPK model obtained, a nonparametric bootstrap resampling method was conducted. One thousand bootstrap resampled data sets were generated, each containing the same number of patients as the original data set, and each of them was fitted individually to the final PPK model. Median values and $95 \%$ confidence intervals for parameter estimates were compared with the parameter estimates obtained from the original data set. Furthermore, to check the suitability of the final model with respect to the observation, a visual predictive check was performed. On the basis of the final PPK model, 1000 plasma concentration profiles following the same dose were simulated without residual errors, and $95 \%$ prediction intervals at each time point were plotted with the observed data.

\section{Statistical analysis}

Statistical analysis was performed with IBM SPSS Statistics version 22 (IBM, Armonk, NY, USA). The relationships between the variables were analyzed by linear regression. A $p$ value of less than 0.05 was considered statistically significant.

\section{Results}

\section{Patient characteristics}

Sixteen patients with CLcr in the range 15.9-108.8 mL/ min were enrolled between December 2013 and November 
Table 1 Patients' characteristics

\begin{tabular}{|c|c|c|c|c|c|}
\hline \multirow[t]{2}{*}{ Characteristics } & \multicolumn{4}{|c|}{ Renal function (CLcr, mL/min) } & \multirow[t]{2}{*}{ Total } \\
\hline & Normal $(\geq 80)$ & Mild (60-79) & Moderate (30-59) & Severe $(<30)$ & \\
\hline No. of patients & 4 & 5 & 5 & 2 & 16 \\
\hline \multicolumn{6}{|l|}{ CLcr (mL/min) } \\
\hline Mean & 97.3 & 67.1 & 48.0 & 22.6 & 63.1 \\
\hline Range & $81.7-108.8$ & $60.7-72.1$ & $40.1-58.2$ & $15.9-29.3$ & $15.9-108.8$ \\
\hline \multicolumn{6}{|c|}{ Serum creatinine $(\mathrm{mg} / \mathrm{dL})$} \\
\hline Mean & 0.91 & 0.87 & 1.04 & 1.48 & 1.00 \\
\hline Range & $0.68-1.39$ & $0.48-1.25$ & $0.61-1.28$ & $1.30-1.66$ & $0.48-1.66$ \\
\hline \multicolumn{6}{|l|}{ Age (years) } \\
\hline Median & 55.5 & 65 & 76 & 71 & 65.5 \\
\hline Range & $46-65$ & $45-78$ & $54-78$ & $66-76$ & $45-78$ \\
\hline \multicolumn{6}{|l|}{ Weight (kg) } \\
\hline Mean & 73.6 & 55.4 & 54.4 & 36.6 & 57.3 \\
\hline Range & $56.5-87.0$ & $42.8-68.3$ & $48.5-64.0$ & $30.2-42.9$ & $30.2-87.0$ \\
\hline \multicolumn{6}{|l|}{$\operatorname{BSA}\left(\mathrm{m}^{2}\right)$} \\
\hline Mean & 1.874 & 1.589 & 1.596 & 1.298 & 1.626 \\
\hline Range & $1.704-2.020$ & $1.328-1.734$ & $1.477-1.801$ & $1.180-1.416$ & $1.180-2.020$ \\
\hline \multicolumn{6}{|l|}{ Sex } \\
\hline Male & 4 & 3 & 3 & 1 & 11 \\
\hline Female & 0 & 2 & 2 & 1 & 5 \\
\hline \multicolumn{6}{|l|}{ PS } \\
\hline 0 & 4 & 4 & 4 & 0 & 12 \\
\hline 1 & 0 & 0 & 1 & 1 & 2 \\
\hline 2 & 0 & 1 & 0 & 1 & 2 \\
\hline \multicolumn{6}{|l|}{ Tumor type } \\
\hline Stomach & 3 & 3 & 5 & 0 & 11 \\
\hline Others & 1 & 2 & 0 & 2 & 5 \\
\hline \multicolumn{6}{|l|}{ Gastrectomy } \\
\hline Yes & 2 & 3 & 4 & 0 & 9 \\
\hline No & 2 & 2 & 1 & 2 & 7 \\
\hline \multicolumn{6}{|c|}{$\mathrm{S}-1$ dose $\left(\mathrm{mg} / \mathrm{m}^{2}\right)$} \\
\hline Mean & 32.1 & 35.4 & 35.1 & 34.6 & 34.4 \\
\hline Range & $29.7-35.2$ & $32.6-37.7$ & $33.3-39.6$ & $33.9-35.3$ & $29.7-39.6$ \\
\hline
\end{tabular}

BSA body surface area, $C L c r$ creatinine clearance estimated by the Cockcroft-Gault equation, $P S$ performance status
2014 (Table 1). The patients were classified into four groups according to renal function as follows: four patients in the normal renal function group (CLcr $\geq 80 \mathrm{~mL} / \mathrm{min}$ ), five patients in the mild dysfunction group (CLcr $=$ 60-79 $\mathrm{mL} / \mathrm{min}$ ), five patients in the moderate dysfunction group $(\mathrm{CLcr}=30-59 \mathrm{~mL} / \mathrm{min})$, and two patients in the severe dysfunction group (CLcr $<30 \mathrm{~mL} / \mathrm{min}$ ).

\section{Pharmacokinetics of 5-FU, CDHP, and tegafur and toxic effects}

The plasma concentration-time profiles of 5-FU, CDHP, and tegafur according to renal function are shown in Fig. 2.
Remarkable increases in the plasma concentrations of 5-FU and CDHP were observed in patients in the severe renal dysfunction group.

No adverse events were observed in any patients within $24 \mathrm{~h}$ of S-1 administration.

Factors influencing the area under the concentration-time curve from 0 to $24 \mathrm{~h}$ of 5-FU

The area under the concentration-time curve (AUC) from 0 to $24 \mathrm{~h}\left(\mathrm{AUC}_{0-24}\right)$ of 5-FU, CDHP, and tegafur calculated with the linear trapezoidal rule in 16 patients was in the 
Fig. 2 Mean ( \pm standard deviation) plasma concentration-time profiles of 5-fluorouracil (5-FU; a), 5-chloro-2,4-dihydroxypyridine $(C D H P ; \mathbf{b})$, and tegafur $(F T ; \mathbf{c})$, according to renal function after a single dose of S-1
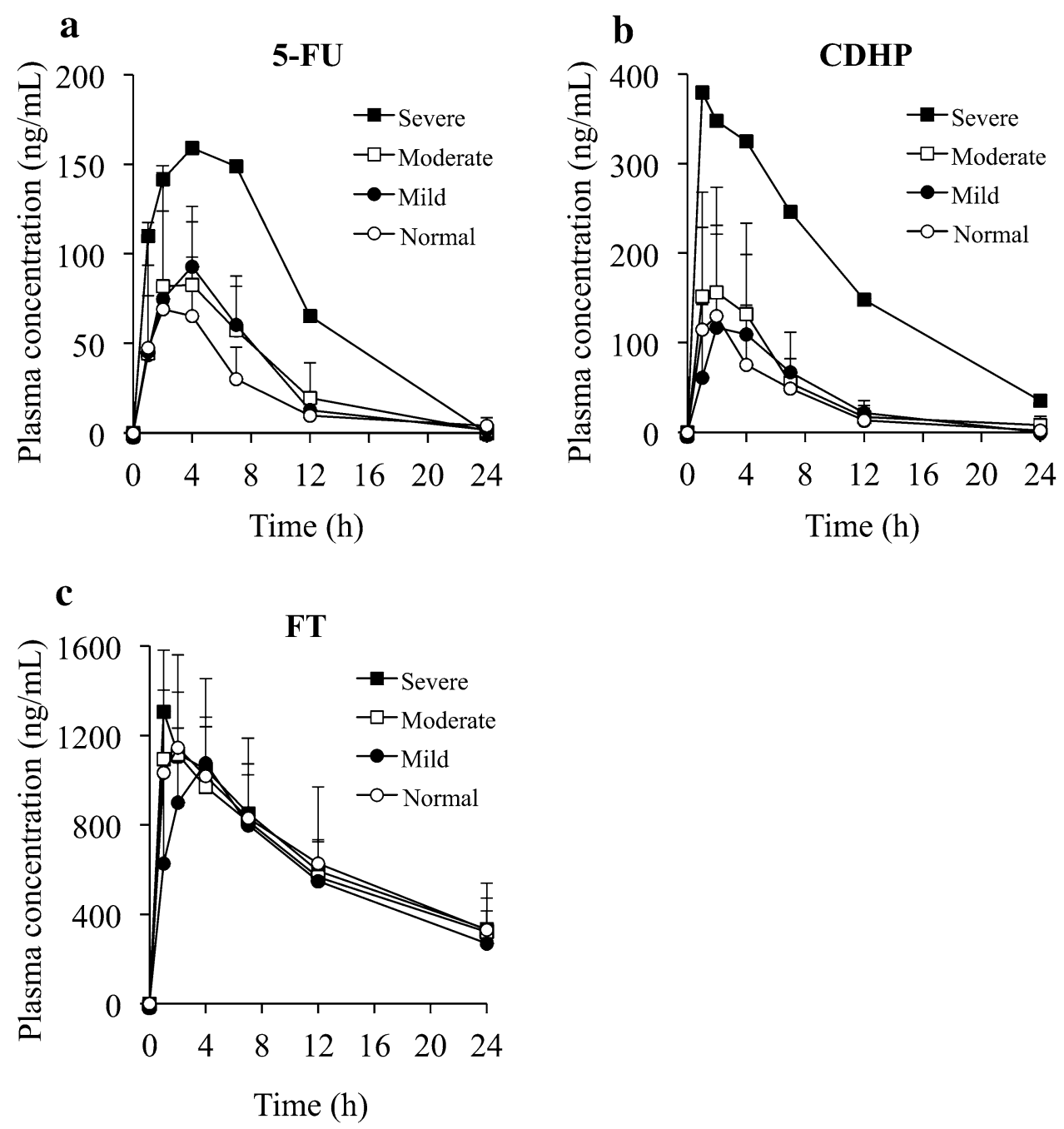

range 286.6-2149.9, 80.4-5295.9, and 8404.9-23403.8 ng $\mathrm{h} / \mathrm{mL}$, respectively. A moderately strong correlation was demonstrated between $\mathrm{AUC}_{0-24}$ of 5-FU and that of CDHP $\left(r^{2}=0.862, p<0.001\right.$; Fig. 3a), whereas $\mathrm{AUC}_{0-24}$ of 5 -FU was not correlated with that of tegafur $\left(r^{2}=0.023\right.$; Fig. 3b). This indicates that exposure of 5-FU is dependent on the exposure of CDHP but not on that of tegafur.

AUC $_{0-24}$ of CDHP correlated moderately with CLcr $\left(r^{2}=0.396, \quad p<0.01 ; \quad\right.$ Fig. $\left.4 a\right), \quad$ and with GFR $\left(r^{2}=0.445, p<0.01\right.$; Fig. $\left.4 \mathrm{~b}\right)$. In addition, $\mathrm{AUC}_{0-24}$ of 5-FU correlated moderately with CLcr $\left(r^{2}=0.458\right.$, $p<0.01$; Fig. $4 \mathrm{c})$ and with GFR $\left(r^{2}=0.416, p<0.01\right.$; Fig. 4d). Both CLcr and GFR could be considered as appropriate measures of renal function, which influenced exposure of both CDHP and 5-FU. We selected CLcr estimated by the Cockcroft-Gault equation as the value of renal function to simulate and predict the pharmacokinetics of 5-FU by PPK analysis because previous reports [14, 20, $21]$ and the summary of product characteristics in Europe
[9] showed and discussed the impact of renal function on S-1 pharmacokinetics with CLcr, but not GFR, as a measure.

\section{PPK analysis of CDHP, 5-FU, and tegafur}

The patients' age, sex, and history of gastrectomy did not have substantial relationships with the individual clearance, volume of distribution, and $K_{\mathrm{a}}$ values of CDHP and 5-FU in the PPK analysis. The individual clearance values of CDHP and 5-FU were related to CLcr and BSA. In addition, the individual volume of distribution values of CDHP and 5-FU were proportional to the BSA. The estimated population mean and variance of the pharmacokinetic parameters of CDHP and 5-FU are shown in Table 2.

The patients' age, sex, history of gastrectomy, renal function, and BSA did not affect the clearance, volume of distribution, and $K_{\mathrm{a}}$ of tegafur in the PPK analysis. The 

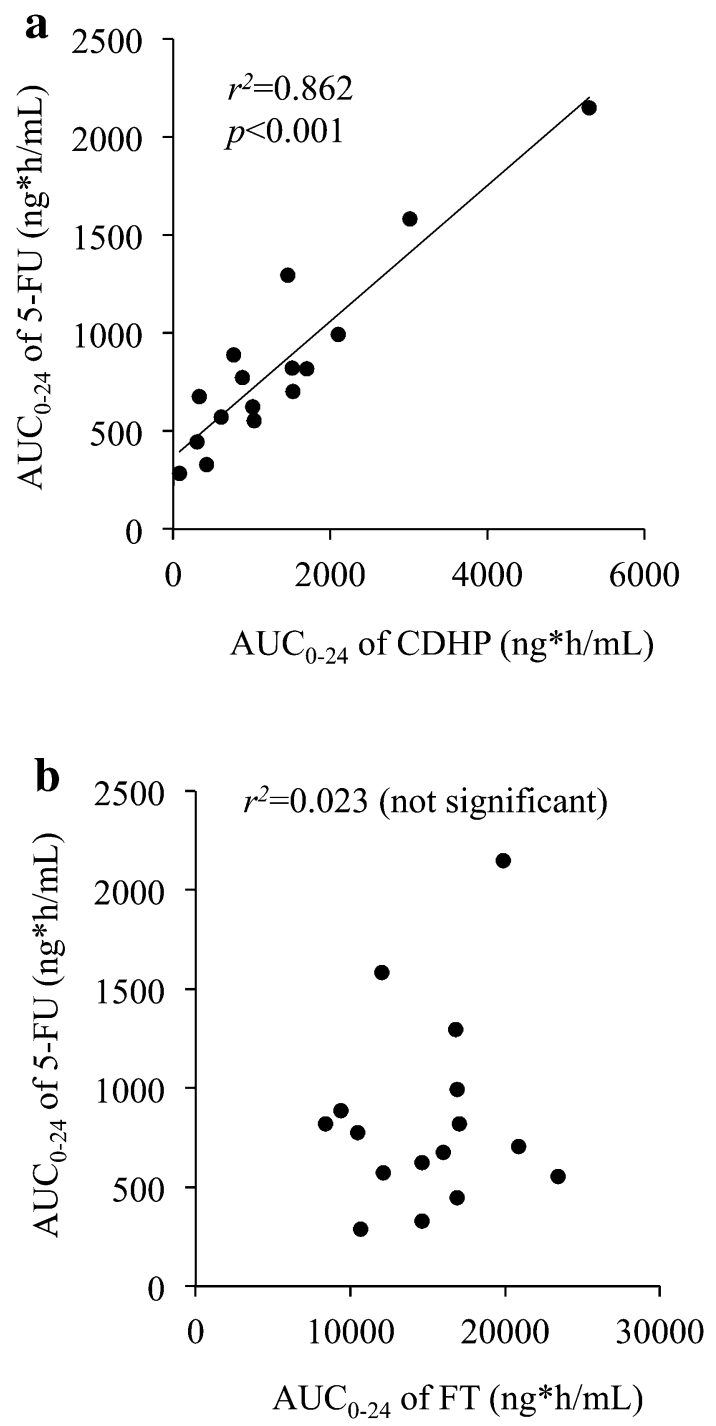

Fig. 3 Correlation between the area under the concentration-time curve from 0 to $24 \mathrm{~h}\left(A U C_{0-24}\right)$ of 5 -fluorouracil $(5-F U)$ and that of 5-chloro-2,4-dihydroxypyridine (CDHP) (a) or tegafur (FT) (b)

estimated population mean and variance of the pharmacokinetic parameters of tegafur are also shown in Table 2.

\section{Model validation}

The results of the visual predictive check and bootstrap validation are presented in Table S1 and Fig. S1. In the bootstrap resampling, more than $99 \%$ of the generated data sets successfully converged. The median values for bootstrap simulation were very consistent with the final parameter estimates obtained with the original data set. These diagnostics and the validation indicated that the final model is robust and reliable for describing the pharmacokinetics of S-1 in patients with various degrees of renal function.

\section{Development of an S-1 dosage formula}

The PPK parameters obtained were used to develop an S-1 dosage formula. The 5-FU clearance related to CLcr and BSA was described by the following equation (Table 2):

$\mathrm{CL} / F=(21.9+0.375 \times \mathrm{CLcr}) \times \mathrm{BSA}$

where $\mathrm{CL}$ is clearance and $F$ is bioavailability. As $\mathrm{AUC}=\mathrm{F} \times$ dose/CL, Eq. 1 was rearranged to

Dose/AUC $=(21.9+0.375 \times$ CLcr $) \times$ BSA

Equation 2 was further rearranged to provide the recommend S-1 dose as follows:

$\begin{aligned} \text { Dose as } \mathrm{FT}= & \text { target } \mathrm{AUC} \text { of } 5-\mathrm{FU} \times(21.9+0.375 \\ & \times \mathrm{CLcr}) \times \mathrm{BSA}\end{aligned}$

The target AUC of 5-FU was derived from previous reports of pivotal registration studies that confirmed the efficacy and toxicity of S-1 at the approved dose. The target AUCs of a single dose were defined as 723.9 and $588.6 \mathrm{ng} \mathrm{h} / \mathrm{mL}$ in Asia (40 mg/m ${ }^{2}$, twice daily) [13] and Europe (25 mg/ $\mathrm{m}^{2}$, twice daily) [22], respectively. The recommended daily doses in Asia and Europe calculated with Eq. 3 are shown as nomograms in Fig. 5 for Asia and Europe with consideration of each approved dosage ( 20 and $25 \mathrm{mg}$ as tegafur in Asia, 15 and $20 \mathrm{mg}$ as tegafur in Europe).

\section{Discussion}

In our study, it was confirmed that renal dysfunction caused high exposure of CDHP and 5-FU in patients with CLcr in the range $15.9-108.8 \mathrm{~mL} / \mathrm{min}$. Ikeda et al. [14] observed increasing exposure of CDHP and 5-FU in rabbits with three degrees of renal dysfunction induced by cisplatin compared with exposure in rabbits with normal renal function. There have been some reports on the relationships between renal function and the AUC of CDHP and/or that of 5-FU in patients with cancer [14, 20, 21]; however, these studies did not include patients with severe renal impairment (CLcr $\geq 36.3 \mathrm{~mL} / \mathrm{min}$ [14], CLcr $\geq 39.0 \mathrm{~mL} / \mathrm{min}$ [20], and CLcr $\geq 54.0 \mathrm{~mL} / \mathrm{min}$ [21]). Therefore, this is the first report to clarify the effect of renal function on the exposure of CDHP and 5-FU in patients with wide variation in renal function, including severe impairment (CLcr $=29.3$ and $15.9 \mathrm{~mL} / \mathrm{min})$.

In patients with CLcr in the range $15.9-108.8 \mathrm{~mL} / \mathrm{min}$, the AUCs of both CDHP and 5-FU were correlated with CLcr and GFR (Fig. 4). Although GFR is widely used to assess renal function for diagnosing kidney disease, it is not always an appropriate measure of renal function for 
Fig. 4 Correlation between the area under the concentrationtime curve from 0 to $24 \mathrm{~h}$ $\left(\mathrm{AUC}_{0-24}\right)$ of 5-chloro-2,4dihydroxypyridine (CDHP) and creatinine clearance (CLcr) (a) or glomerular filtration rate (GFR) (b). Correlation between $\mathrm{AUC}_{0-24}$ of 5-fluorouracil (5FU) and CLcr (c) or GFR (d). CLcr and GFR were estimated by the Cockcroft-Gault equation and the Japanese GFR equation, respectively

\section{a}

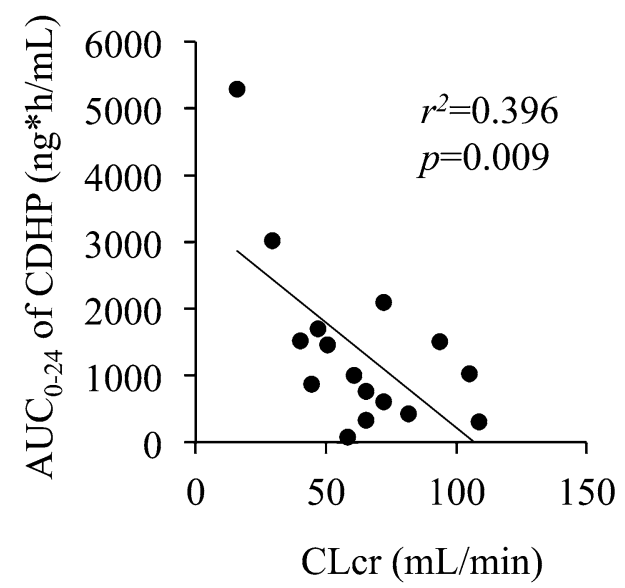

c

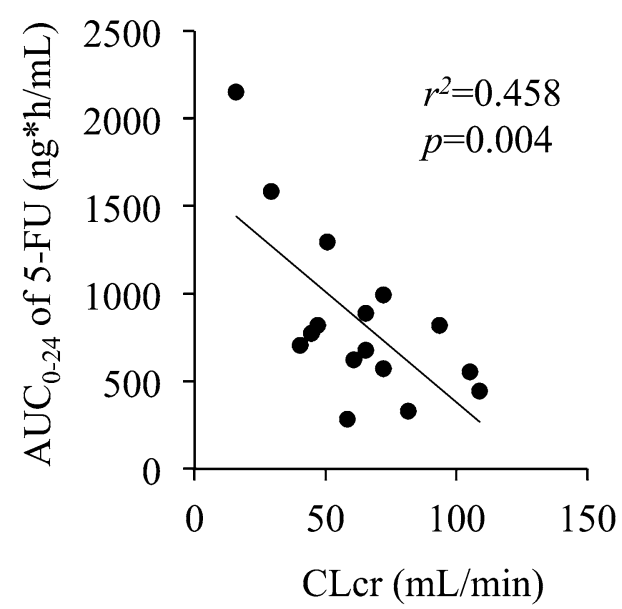

b

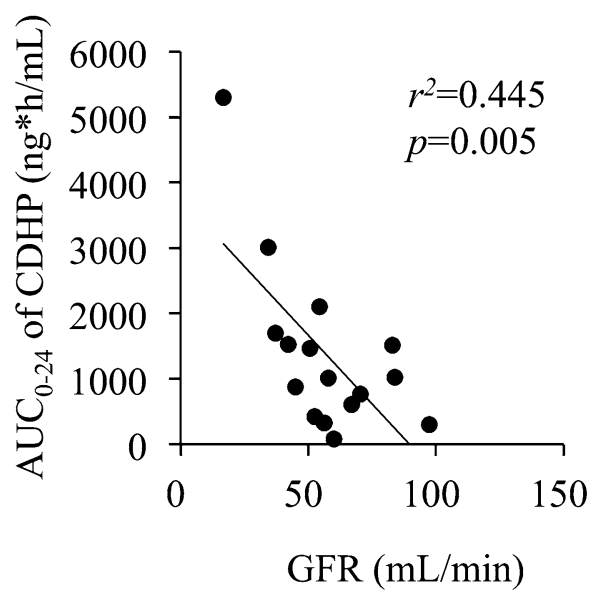

d

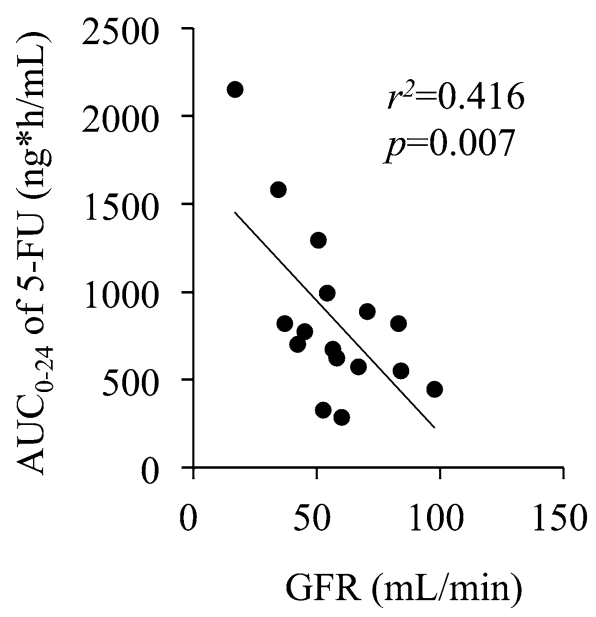

Table 2 Estimated parameters of the population pharmacokinetic model

\begin{tabular}{llll}
\hline Compound & Parameter & Population mean & Interindividual variability $(\%)$ \\
\hline 5-FU & $K_{\mathrm{a}}(1 / \mathrm{h})$ & 0.551 & 202 \\
& $\mathrm{CL} / F\left(\mathrm{~L} / \mathrm{h} / \mathrm{m}^{2}\right)$ & $(21.9+0.375 \times \mathrm{CLcr}) \times \mathrm{BSA}$ & 27.5 \\
& $V / F\left(\mathrm{~L} / \mathrm{m}^{2}\right)$ & $362 \times \mathrm{BSA}$ & 87.7 \\
& Residual variability $(\%)$ & 37.9 & \\
CDHP & $K_{\mathrm{a}}(1 / \mathrm{h})$ & 1.04 & 167 \\
& $\mathrm{CL} / F\left(\mathrm{~L} / \mathrm{h} / \mathrm{m}^{2}\right)$ & $(3.77+0.403 \times \mathrm{CLcr}) \times \mathrm{BSA}$ & 49.9 \\
& $V / F\left(\mathrm{~L} / \mathrm{m}^{2}\right)$ & $200 \times \mathrm{BSA}$ & 79.0 \\
& Residual variability $(\%)$ & 33.3 & 121 \\
Tegafur & $K_{\mathrm{a}}(1 / \mathrm{h})$ & 1.46 & 41.8 \\
& $\mathrm{CL} / F\left(\mathrm{~L} / \mathrm{h} / \mathrm{m}^{2}\right)$ & 2.89 & 23.6 \\
& $V / F\left(\mathrm{~L} / \mathrm{m}^{2}\right)$ & 44.9 & \\
\hline
\end{tabular}

$B S A$ body surface area, $C D H P$ 5-chloro-2,4-dihydroxypyridine, $C L$ clearance, $C L c r$ creatinine clearance estimated by the Cockcroft-Gault equation, $F$ bioavailability, 5- $F U$ fluorouracil, $K_{a}$ first-order absorption rate constant, $V$ volume of distribution 
predicting drug exposure and adjusting the drug dosage in patients with renal impairment. Renal drug elimination comprises the combined processes of glomerular filtration, tubular secretion, and reabsorption. Putt et al. [23] demonstrated that measured GFR approximated renal tubular anion excretion and reabsorption, but did not correlate well with cationic drug transport. Furthermore, they suggested that dose adjustments based on GFR may underestimate clearances and potentially mislead the clinician to prescribe ineffective doses of important drugs eliminated via tubular pathways. This finding is very important for future discussion of our study, although the route of CDHP renal elimination remains unclear.

An S-1 dosage formula based on renal function as indicated by CLcr was derived according to the concept of exposure matching to the approved dose of S-1 in pivotal registration studies (Eq. 3). Systemic exposure is one of the indirect pharmacokinetic measures for product quality bioequivalence [24, 25]. The US Food and Drug Administration and the European Medicines Agency also recommend dose adjustment to produce a range of plasma concentrations of drugs or active metabolites that is similar in subjects with normal renal function and those with impaired renal function [1,2]. Our S-1 dosage formula was developed by PPK analysis in 16 patients with various degrees of CLcr in the range $15.9-108.8 \mathrm{~mL} / \mathrm{min}$. The number of patients (16) is not insufficient to derive the initial formula by reference to the development of a carboplatin dosage formula by Calvert et al. [26]. They derived the initial formula from a retrospective analysis of carboplatin pharmacokinetics in only 18 patients with GFR in the range $33-136 \mathrm{~mL} / \mathrm{min}$, and evaluated it in 31 patients prospectively. The reliability of the formula we have developed must be evaluated subsequently for practical application.

We have proposed an original formula (Eq. 3) and nomograms (Fig. 5) illustrating the recommended dose of S-1 according to patients' CLcr and BSA in consideration of the dosage (20 and $25 \mathrm{mg}$ as tegafur in Asia, 15 and $20 \mathrm{mg}$ as tegafur in Europe). On the Asian package insert, the dosage and administration are presented only for patients with normal renal function [7, 8]. Therefore, this is the first information on dose optimization for patients with impaired renal function in Asia. Furthermore, regarding dose modification, the European summary of product characteristics indicates that the standard dose should be administered without modification in patients with mild renal impairment $(\mathrm{CLcr}=51-80 \mathrm{~mL} / \mathrm{min})$, whereas the drug should be administered at $40 \mathrm{mg} / \mathrm{m}^{2} /$ day as tegafur for patients with moderate renal impairment $(\mathrm{CLcr}=$ $30-50 \mathrm{~mL} / \mathrm{min}$ ) [9]. These values were derived from a Monte Carlo simulation of virtual patients with renal dysfunction using a previously developed PPK model for S-1
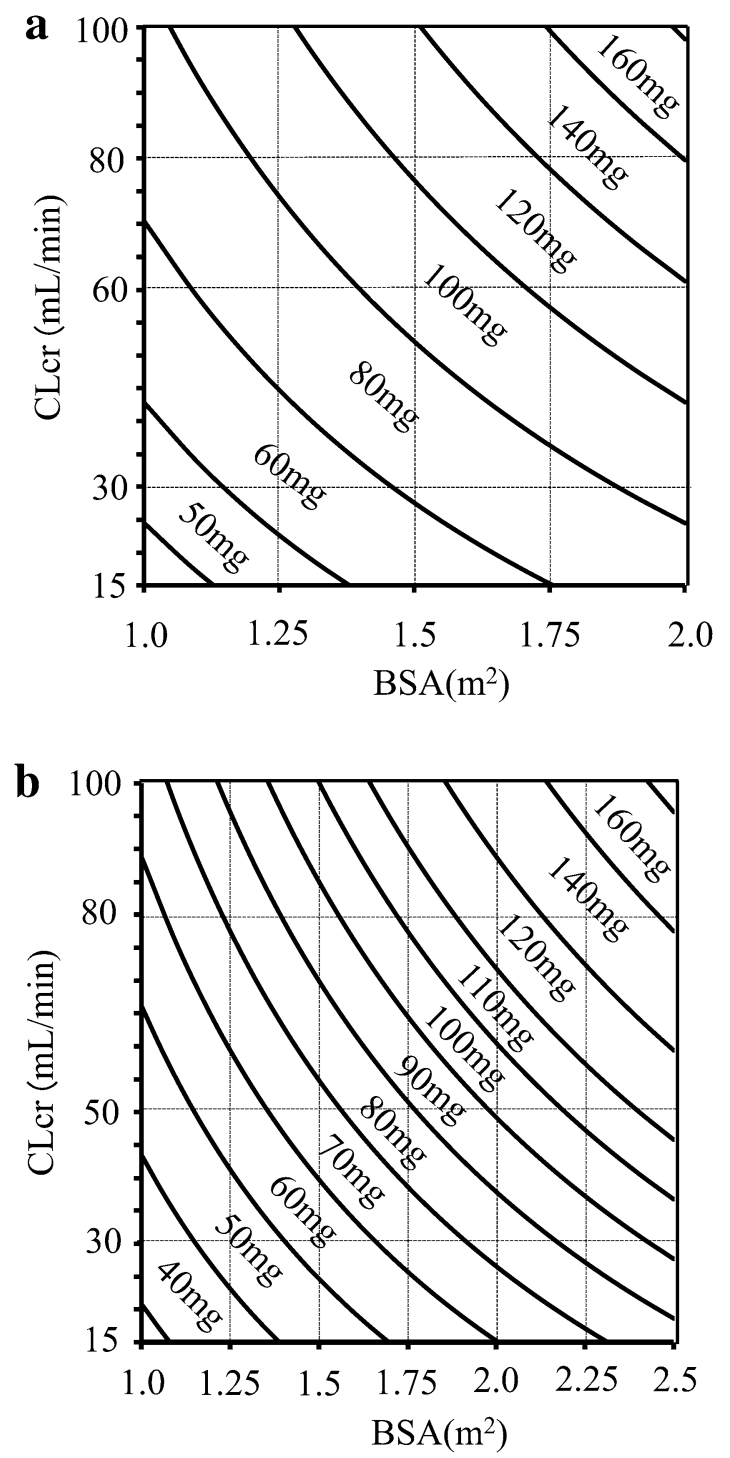

Fig. 5 Recommended total daily doses of S-1 as tegafur, according to creatinine clearance $(C L c r)$ estimated by the Cockcroft-Gault equation and body surface area $(B S A)$ in Asia for the approved dose of $80 \mathrm{mg} / \mathrm{m}^{2}$ as tegafur (a) and in Europe for the approved dose of $50 \mathrm{mg} / \mathrm{m}^{2}$ as tegafur (b)

in Western patients [27]. The limited guidance on S-1 dose adjustment in the summary of product characteristics is similar to the recommended dose provided by the formula we have developed. For patients with severe renal impairment, our formula also provides a novel dosing recommendation for S-1 in Europe.

Tegafur is a prodrug of 5-FU with good oral bioavailability. Following oral administration, tegafur is gradually converted to 5-FU, mainly by cytochrome P450 (CYP) 2A6 [28, 29]. Although CYP2A6 variants are associated with the pharmacokinetic variability of tegafur [30], the AUC of CDHP, which is affected by renal function, is the key determinant of the pharmacokinetic variability of 5-FU 
[20]. Generally, prior gastrectomy causes a change in the absorption of orally administered drugs. In our 16 patients, including nine patients who had previously undergone gastrectomy, a history of gastrectomy did not affect the individual pharmacokinetics of tegafur and CDHP according to the PPK analysis. The resulting exposure of 5-FU was not influenced by gastrectomy. The effect of gastrectomy on the pharmacokinetics of 5-FU after S-1 administration is unclear $[19,31-33]$. However, as approximately only $10 \%$ of absorbed tegafur is converted to 5-FU in terms of the plasma concentrations of tegafur and 5-FU, we do not consider a history of gastrectomy to be a crucial factor affecting the pharmacokinetics of 5-FU. Therefore, the formula we have developed is expected to apply for patients regardless of CYP2A6 polymorphism, which causes ethnic differences according to race-related differences in the allele frequency, and a history of gastrectomy.

In conclusion, we have developed a simple formula for determining the S-1 dosage on the basis of individual CLcr and BSA values. The recommended daily doses of S-1 in Asia and Europe were also proposed as nomograms according to each approved dose and dosage. Further validation is needed to confirm the formula for practical application.

Acknowledgments The authors thank the patients and their families for their participation in this study.

\section{Compliance with the ethical standards}

Conflict of interest Narikazu Boku has received honoraria and research funding from Taiho Pharmaceutical. Yusuke Tanigawara has served as a consultant for Taiho Pharmaceutical and Meiji Seika Pharma. Yuko Kitagawa has received grants from Otsuka Pharmaceutical, Taiho Pharmaceutical, Takeda Pharmaceutical, and Nippon Kayaku, and is a member of the speakers bureau for Taiho Pharmaceutical and Nippon Kayaku. The other authors declare that they have no conflict of interest.

Ethical standards All procedures followed were in accordance with the ethical standards of the responsible committee on human experimentation (institutional and national) and with the Helsinki Declaration of 1964 and later versions. Informed consent was obtained from all patients for their being included in the study.

Funding This work was not supported by any direct or indirect funding.

Open Access This article is distributed under the terms of the Creative Commons Attribution 4.0 International License (http://crea tivecommons.org/licenses/by/4.0/), which permits unrestricted use, distribution, and reproduction in any medium, provided you give appropriate credit to the original author(s) and the source, provide a link to the Creative Commons license, and indicate if changes were made.

\section{References}

1. US Food Drug Administration. Guidance for industry: pharmacokinetics in patients with impaired renal function — study design, data analysis, and impact on dosing and labeling. 2010. http:// www.fda.gov/downloads/Drugs/GuidanceComplianceRegulator yInformation/Guidances/ucm072127.pdf. Accessed 21 July 2015.

2. European Medicines Agency. Note for guidance on the evaluation of the pharmacokinetics of medical products in patients with renal function. 2004. http://www.ema.europa.eu/docs/en_GB/ document_library/Scientific_guideline/2009/09/WC500003123.pdf. Accessed 21 July 2015.

3. Heidelberger C, Chaudhuri NK, Danneberg P, Mooren D, Griesbach L, Duschinsky R, et al. Fluorinated pyrimidines, a new class of tumour-inhibitory compounds. Nature. 1957;179:663-6.

4. Shirasaka T, Nakano K, Takechi T, Satake H, Uchida J, Fujioka A, et al. Antitumor activity of $1 \mathrm{M}$ tegafur- $0.4 \mathrm{M}$ 5-chloro-2,4dihydroxypyridine-1 M potassium oxonate (S-1) against human colon carcinoma orthotopically implanted into nude rats. Cancer Res. 1996;56:2602-6.

5. Shirasaka T, Shimamato Y, Ohshimo H, Yamaguchi M, Kato T, Yonekura K, et al. Development of a novel form of an oral 5 -fluorouracil derivative (S-1) directed to the potentiation of the tumor selective cytotoxicity of 5-fluorouracil by two biochemical modulators. Anticancer Drugs. 1996;7:548-57.

6. Shirasaka T, Shimamoto Y, Fukushima M. Inhibition by oxonic acid of gastrointestinal toxicity of 5-fluorouracil without loss of its antitumor activity in rats. Cancer Res. 1993;53:4004-9.

7. Taiho Pharmaceutical. TS-1 prescribing information in Japan; TS-1 combination OD tablet. Ver. 4; July 2014.

8. Taiho Pharmaceutical. TS-1 prescribing Information in Singapore. Revised: TS-ONE capsule; 2013.

9. European Medicines Agency. Teysuno : EPAR - product information. 2013. http://www.ema.europa.eu/docs/en_GB/document_ library/EPAR___Product_Information/human/001242/WC500104415. pdf. Accessed 21 July 2015.

10. Sakuramoto S, Sasako M, Yamaguchi T, Kinoshita T, Fujii M, Nashimoto A, et al. Adjuvant chemotherapy for gastric cancer with S-1, an oral fluoropyrimidine. N Engl J Med. 2007;357: 1810-20.

11. Boku N, Yamamoto S, Fukuda H, Shirao K, Doi T, Sawaki A, et al. Fluorouracil versus combination of irinotecan plus cisplatin versus S-1 in metastatic gastric cancer: a randomised phase 3 study. Lancet Oncol. 2009;10:1063-9.

12. Koizumi W, Narahara H, Hara T, Takagane A, Akiya T, Takagi $\mathrm{M}$, et al. S-1 plus cisplatin versus S-1 alone for first-line treatment of advanced gastric cancer (SPIRITS trial): a phase III trial. Lancet Oncol. 2008;9:215-21.

13. Hirata K, Horikoshi N, Aiba K, Okazaki M, Denno R, Sasaki K, et al. Pharmacokinetic study of S-1, a novel oral fluorouracil antitumor drug. Clin Cancer Res. 1999;5:2000-5.

14. Ikeda M, Furukawa $H$, Imamura $H$, Shimizu J, Ishida $H$, Masutani S, et al. Pharmacokinetic study of S-1, a novel oral fluorouracil antitumor agent in animal model and in patients with impaired renal function. Cancer Chemother Pharmacol. 2002;50:25-32.

15. Cockcroft DW, Gault MH. Prediction of creatinine clearance from serum creatinine. Nephron. 1976;16:31-41.

16. US Department of Health and Human Services. Common Terminology Criteria for Adverse Events (CTCAE) version 4.0. 2010. http://evs.nci.nih.gov/ftp1/CTCAE/CTCAE_4.03_2010-0614_QuickReference_5x7.pdf. Accessed 21 July 2015. 
17. Matsuo S, Imai E, Horio M, Yasuda Y, Tomita K, Nitta K, et al. Revised equations for estimated GFR from serum creatinine in Japan. Am J Kidney Dis. 2009;53:982-92.

18. Liu K, Zhong D, Zou H, Chen X. Determination of tegafur, 5-fluorouracil, gimeracil and oxonic acid in human plasma using liquid chromatography-tandem mass spectrometry. J Pharm Biomed Anal. 2010;52:550-6.

19. Hirose T, Fujita K, Nishimura K, Ishida H, Yamashita K, Sunakawa Y, et al. Pharmacokinetics of S-1 and CYP2A6 genotype in Japanese patients with advanced cancer. Oncol Rep. 2010;24: 529-36.

20. Fujita K, Yamamoto W, Endo S, Endo H, Nagashima F, Ichikawa $\mathrm{W}$, et al. CYP2A6 and the plasma level of 5-chloro-2,4-dihydroxypyridine are determinants of the pharmacokinetic variability of tegafur and 5-fluorouracil, respectively, in Japanese patients with cancer given S-1. Cancer Sci. 2008;99:1049-54.

21. Ando Y, Kawada K, Inada M, Morita S, Mitsuma A, Yasuda Y, et al. Pharmacokinetic study of S-1 in patients in whom inulin clearance was measured. Oncology. 2012;83:38-44.

22. van Groeningen CJ, Peters GJ, Schornagel JH, Gall H, Noordhuis $\mathrm{P}$, de Vries MJ, et al. Phase I clinical and pharmacokinetic study of oral S-1 in patients with advanced solid tumors. J Clin Oncol. 2000;18:2772-9.

23. Putt TL, Duffull SB, Schollum JB, Walker RJ. Gfr may not accurately predict aspects of proximal tubule drug handling. Eur J Clin Pharmacol. 2014;70:1221-6.

24. US Food Drug Administration. Guidance for industry: bioavailability and bioequivalence studies for orally administered drug products - general considerations. 2003. http://www.fda.gov/ downloads/Drugs/Guidances/ucm070124.pdf. Accessed 21 July 2015.

25. European Medicines Agency. Guideline on the investigation of bioequivalence. 2010. http://www.ema.europa.eu/docs/en_GB/
document_library/Scientific_guideline/2010/01/WC500070039. pdf. Accessed 21 July 2015.

26. Calvert AH, Newell DR, Gumbrell LA, O'Reilly S, Burnell M, Boxall FE, et al. Carboplatin dosage: prospective evaluation of a simple formula based on renal function. J Clin Oncol. 1989;7: 1748-56.

27. Comets E, Ikeda K, Hoff P, Fumoleau P, Wanders J, Tanigawara Y. Comparison of the pharmacokinetics of S-1, an oral anticancer agent, in Western and Japanese patients. J Pharmacokinet Pharmacodyn. 2003;30:257-83.

28. Ikeda K, Yoshisue K, Matsushima E, Nagayama S, Kobayashi K, Tyson CA, et al. Bioactivation of tegafur to 5-fluorouracil is catalyzed by cytochrome P-450 2A6 in human liver microsomes in vitro. Clin Cancer Res. 2000;6:4409-15.

29. Komatsu T, Yamazaki H, Shimada N, Nakajima M, Yokoi T. Roles of cytochromes P450 1A2, 2A6, and 2C8 in 5-fluorouracil formation from tegafur, an anticancer prodrug, in human liver microsomes. Drug Metab Dispos. 2000;28:1457-63.

30. Ichikawa W, Fujita K, Sasaki Y. The unanswered question: what is the determinant of S-1 pharmacokinetics? Clin Pharmacol Ther. 2008;84:203; author reply 204.

31. Kim WY, Nakata B, Hirakawa K. Alternative pharmacokinetics of S-1 components, 5-fluorouracil, dihydrofluorouracil and alphafluoro-beta-alanine after oral administration of S-1 following total gastrectomy. Cancer Sci. 2007;98:1604-8.

32. Kochi M, Fujii M, Kanamori N, Kaiga T, Aizaki K, Takahashi T, et al. Effect of gastrectomy on the pharmacokinetics of S-1, an oral fluoropyrimidine, in resectable gastric cancer patients. Cancer Chemother Pharmacol. 2007;60:693-701.

33. Tsuruoka Y, Kamano T, Kitajima M, Kawai K, Watabe S, Ochiai $\mathrm{T}$, et al. Effect of gastrectomy on the pharmacokinetics of 5-fluorouracil and gimeracil after oral administration of S-1. Anticancer Drugs. 2006;17:393-9. 